\title{
SUR LES POUVOIRS THERMOÉLECTRIQUES DE QUELQUES OXYDES ET SULFURES MÉTALLIQUES;
}

Par M. Eny. VAx AUBEL.

Anton Abt a publié, dans le $\mathrm{n}^{\circ} 6$ de l'année 1900 de Drude's Annalen der Physik, un mémoire sur les forces électromotrices thermoélectriques de quelques oxydes et sulfures métalliques.

Ce physicien a étudié les forces électromotrices thermoélectriques au moyen de la méthode de compensation de Poggendorff, modifiée par Kirchhoff. Si $e$ et $e^{\prime}$ sont les forces électromotrices de deux éléments thermoélectriques à comparer, on a : $\frac{e}{e^{\prime}}=\frac{a}{a^{\prime}}$, $a$ et $a^{\prime}$ représentant les longueurs correspondantes d'un fil de platine tendu, pour lesquelles la compensation est réalisée. Cela étant, voici quelques valeurs obtenues pour la longueur $a$, qui est proportionnelle à la force thermoélectrique du couple considéré; nous allons devoir les utiliser.

\begin{tabular}{|c|c|c|}
\hline & Couple thermoélectriqur. & Valeur de $a$ \\
\hline Bismuth & et $\operatorname{zinc} \ldots \ldots \ldots \ldots \ldots$ & 134,6 \\
\hline - & et antimoine........... & 199,7 \\
\hline Pyrolusi & te et bismuth.......... & 210,6 \\
\hline - & et nickel............ & $28 \mathrm{a}, 1$ \\
\hline - & et charbon........... & $31 \breve{3,2}$ \\
\hline - & et zinc.............. & 331,1 \\
\hline - & et $\mathrm{fer} \ldots \ldots \ldots \ldots \ldots$ & $3 \% 3,9$ \\
\hline - & et pyrrhotite...$\ldots \ldots$. & 370,1 \\
\hline - & et antimoine $\ldots \ldots \ldots$. & 396,9 \\
\hline Pyrihoti & te et fer............... & 11,0 \\
\hline$\cdots$ & et antimuine $\ldots \ldots \ldots$. & 21,7 \\
\hline - & et zinc $\ldots \ldots \ldots \ldots \ldots$ & 29,7 \\
\hline 一 & et nickel........... & 39,8 \\
\hline - & et bismuth $\ldots \ldots \ldots \ldots$. & 178,0 \\
\hline - & et chalcopyrite ........ & $6 \mathfrak{4} 9, \mathfrak{\jmath}$ \\
\hline Chalcopy & rite et bismuth. . . . . . . . & 342,2 \\
\hline - & et zinc. ..... & 602,6 \\
\hline - & et nickel.......... & 618,8 \\
\hline 一 & et fer........... & $66 \pi, 1$ \\
\hline - & et antimoine $\ldots \ldots \ldots$ & 669,3 \\
\hline
\end{tabular}




\begin{tabular}{|c|c|c|}
\hline Pyrite & et pyrrhotite..... & 563,2 \\
\hline - & et antimoine $\ldots \ldots \ldots \ldots$ & 576,3 \\
\hline - & et $\operatorname{zinc} \ldots \ldots \ldots \ldots \ldots \ldots$ & 600,5 \\
\hline - & et charbon............... & 615,6 \\
\hline - & et fer............. & $63 \check{a}, 9$ \\
\hline - & et nickel .. & 642,9 \\
\hline - & et bismuth............. & 731,9 \\
\hline - & et pyrolusite $\ldots \ldots \ldots \ldots \ldots$ & 933,2 \\
\hline
\end{tabular}

Les diverses valeurs de $a$ ont permis, à l'auteur du mémoire qui nous occupe, de formuler les conclusions suivantes (page 278):

"Vergleicht man die milgeteilten Resultaten mit einander, so " erhält man für die von mir untersuchten Metallulfide und Metal"loxycle folgendle Spannungsreihe:

Chalkopyrit
Pyrolusit
Wismut
Zink
Nickel

Kupfer

Cadmium

Nickelerz

Kohle

(für Bogenlicht)

Eisen
Pyrrhotit
Antimon
Pyrit
+

"Die Glender dieser Reihe folgen dem Gesetze der thermoelehtrischen "Spannungsreihe der Metalle mit hinreichender Annäherung. So " ist z. $B$ :

$$
\begin{aligned}
& \text { Pyrolusit } / \text { Kohle }+ \text { Kohle } / \text { Pyrit }=\text { Pyrolusit } / \text { Pyrit } \\
& 315,2+61 \text { o }, 6=930,8 \text { (beob. } 933,2 \text { ), }
\end{aligned}
$$

ferner

$$
\begin{array}{cccc}
\text { Wismut } / \text { Zink } & + & \text { Zink/Pyrit } & =\text { Wismut/Pyrit } \\
134,6 & + & 600,5 & =73 \check{5}, 1 \text { (beob. 731,9). }
\end{array}
$$

Je me suis proposé de vérifier cette dernière conclusion de A. Abt, sur d'autres exemples pris parmi les résultats de ses mesures. Constatons tout d'abord que ce physicien trouve:

\begin{tabular}{|c|c|}
\hline Couple & chalcopyrite/fer............ \\
\hline- & chalcopyrite/zinc....... \\
\hline- & pyrrhotite/zinc........... \\
\hline - & 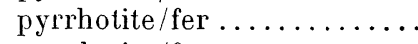 \\
\hline - & pyrolusite $/$ fer ............ \\
\hline - & pyrolusite $/$ zinc $\ldots \ldots \ldots \ldots \ldots$ \\
\hline
\end{tabular}

$$
\begin{aligned}
& \text { Pour le couple pyrite/zinc....................... } a=600,5 \\
& \text { pyrite/fer.............. } a=63 \dddot{3}, 9
\end{aligned}
$$

Le fer devrait donc, dans la série thermoélectrique, être plus éloigné de la pyrite que le zinc. Mais on a, d'autre part : 
Ces trois dernières séries de résultats établissent, au contraire, que le zinc et le fer occupent, dans la série des pouvoirs thermoélectriques, les places qui leur ont été assignées par A. Abt. D'autres cas analogues se présentent. Ainsi les valeurs obtenues pour les couples pyrrhotite/nickel et pyrrhotite/zinc, d'une part, pyrite/fer et pyrite /zinc, d'autre part, conduisent à donner au nickel, au zine et au fer des positions dans la série des pouvoirs thermoélectriques, qui sont autres que celles qui leur ont été attribuées.

Calculons, maintenant, les valeurs de la force électromotrice des couples nickel/fer, zinc/fer, bismuth/antimoine et bismuth/pyrrhotite, en faisant la somme ou la différence des forces électromotrices de couples convenablement choisis. Nous obtenons ainsi les résultats suivants, pour les diverses valeurs de la longueur $a$. :

Couple nickel,fer.

1. (Pyrite $/$ nickel $)-($ pyrite $/$ fer $)=$ nickel $/$ fer.

ou $642,9 \quad-635,9=7,0$

2. (Chalcopyrite/fer) - (chalcopyrite/nickel) $=$ nickel ${ }^{\text {fer. }}$

ou $667,1 \quad-\quad 618,8 \quad=48,3$

3. $($ Pyrrhotite $/$ nickel $)-($ pyrrhotite $/$ fer $)=$ nickel $/$ fer.

$\begin{array}{llll}\text { ou } & 59,8 \quad-\quad 11,0 & - & 48,8\end{array}$

4. Pyrolusite $/$ fer $)-($ pyrolusite $/$ nickel $)=$ nickel $/$ fer.

ou $\quad 3 \ddot{3} 3,9-28 \%, 1$ -

Ainsi la longueur $a$ est, suivant les cas, égale à $7,0-48,3-\mathbf{4 8 , 8}$ et 68,8 .

Couple zincifer.

1. (Chalcopyrite/fer $)-($ chalcopyrite zinc $)=$ (zinc fer $)$.

ou $667,1 \quad-602,6=64,5$

2. $($ Pyrrhotite/zinc) - (pyrrhotite fer $=$ zinc fer $)$.

ou $29,7 \quad-\quad 11,0=18$, ;

3. $($ Pyrolusite $/$ fer $)-($ pyrolusite zinc $)=($ zinc $/$ fer $)$.

ou $353,9-331,1=22,8$

La longueur $a$ varie donc de 18,7 à 22,8 à 64, ŏ.

Couple bismuth/antimoine.

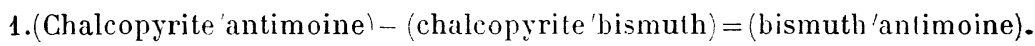

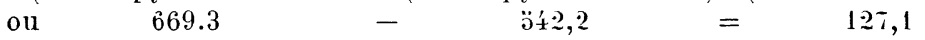

2. (Pyrite'bismuth) - (pyrite antimoine $)=$ bismuth antimoine.

ou $\quad 713,9 \quad-\quad \check{3} 76,3=1.30,6$ 
3. (Pyrrhorite $/$ bismuth $)+$ (pyrrhotite/antimoine $)=($ bismuth/antimoine $)$. ou $178,0+21,7 \quad=199,7$

4. (Pyrolusite $/$ antimoine $)$ - (pyrolusite/bismuth $)=$ (bismuth/antimoine $)$.

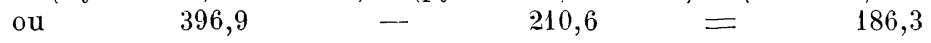

La longueur $a$ est, suivant les cas, 12ఫ,1 - 1əั5,6 - 186,3-199,7. L'étude directe du couple bismuth/antimoine a donné à A. Abt: $a=199, \overline{7}$.

Couple pyrrhotite/bismuth.

1. (Pyrite $/$ bismuth $)-($ pyrite/pyrrhotite $)=($ pyrrhotite $/$ bismuth $)$. ou $731,9 \quad-\quad 353,2 \quad=\quad 168,7$

2.(Chalcopyrite/pyrrhotite $)-($ chalcopyrite $/$ bismuth $)=($ pyrrhotite $/$ bismuth $)$. ou $649, \because 3 \quad 542,2 \quad=107,3$

3. (Pyrolusite/pyrrhotite $)$ - (pyrolusite $/$ bismuth $)=($ pyrrhotite $/$ bismuth $)$. $\begin{array}{lllll}\text { ou } & 370,1 \quad 210,6 \quad & = & 159,0\end{array}$

La longueur a est ici 107,3 - 15̆9,oั ou 168,7, d'après les couples thermoélectriques considérés. La mesure directe, pour le couple pyrrhotite / bismuth, avait conduit à : $a=178,0$.

Il est inutile de multiplier davantage les exemples, pour démontrer que la conclusion du mémoire de A. Abt, rappelée plus haut, ne se vérifie nullement et pour établir que les corps étudiés par lui ne suivent pas la loi, à laquelle obéit la série des pouvoirs thermoélectriques des mélaux. 